\title{
Dialogic feedback
}

David Carless

\section{Source}

Carless, David. (2013). Trust and its role in facilitating dialogic feedback. Feedback in higher and professional education: Understanding it and doing it well.

Dialogic feedback [is defined] as interactive exchanges in which interpretations are shared, meanings negotiated and expectations clarified ... dialogic feedback is facilitated when teachers and students enter into trusting relationships in which there are ample opportunities for interaction about learning and the notions of quality (p. 90). 